\title{
Elasticity of jammed packings of sticky disks
}

\author{
Dion J. Koeze, Lingtjien Hong $\odot$, Abhishek Kumar, and Brian P. Tighe ${ }^{\circ}$ \\ Process \& Energy Laboratory, Delft University of Technology, Leeghwaterstraat 39, 2628 CB Delft, The Netherlands
}

(Received 28 January 2020; revised 28 May 2020; accepted 3 August 2020; published 21 August 2020)

\begin{abstract}
Numerous soft materials jam into an amorphous solid at a high packing fraction. This nonequilibrium phase transition is best understood in a model system where particles repel when they overlap. Recently, however, it was shown that introducing any finite amount of attraction between particles changes the universality class of the transition. The properties of this "sticky jamming" class remain almost entirely unexplored. We use molecular dynamics simulations and scaling analysis to determine the shear modulus, bulk modulus, and coordination of marginal solids close to the sticky jamming point. Each observable differs not just quantitatively but also qualitatively from the purely repulsive case.
\end{abstract}

DOI: 10.1103/PhysRevResearch.2.032047

Non-Brownian dispersions, including emulsions, foams, and pastes, jam into amorphous solids above a critical packing fraction [1]. Nearly all numerical and theoretical studies of the jamming transition employ a (by now canonical) model where athermal spheres repel when they overlap [2-4]. Nevertheless, soft matter generically displays some degree of "stickiness," e.g., due to depletion interactions in emulsions [5-7], finite contact angles in foams [8], or liquid bridges in wet granular media [9-12] and capillary suspensions [13].

We expect the jamming scenario for sticky particles to be relevant to a broad range of natural and engineered systems where attraction is generically present. Nevertheless, many of the fundamental mechanical and structural properties of sticky jammed matter remain unexplored. The relatively few studies of sticky soft spheres that are available reveal important differences from repulsive jamming: (i) Sticky particles jam at lower packing fractions, with structural signatures reminiscent of gels $[14,15]$; (ii) they form shear bands under conditions where repulsive particles do not [16-19]; and (iii) most tellingly, they belong to a distinct universality class [20]. In fact, any finite attraction between particles places a system in the sticky jamming class [21].

What distinguishes sticky jammed solids from their repulsive counterparts? In repulsive jamming, the elastic moduli and mean coordination display power-law scaling as a function of distance to the critical packing fraction [3,4]. While it seems plausible that similar scaling relations exist near sticky jamming, this hypothesis needs to be tested. Here, we study critical scaling in marginally jammed packings of sticky disks [Fig. 1(a)] and show that they depart qualitatively from the repulsive jamming scenario in three distinct ways. First, the shear modulus $G$ vanishes with a critical exponent that is

Published by the American Physical Society under the terms of the Creative Commons Attribution 4.0 International license. Further distribution of this work must maintain attribution to the author(s) and the published article's title, journal citation, and DOI. much larger than its repulsive counterpart. Second, the bulk modulus $K$ also vanishes continuously at the sticky jamming point, unlike the discontinuous transition seen in repulsive systems. Finally, constraints on motion (i.e., bonds) outnumber particulate degrees of freedom at the sticky jamming transition. Balance between the two, or isostaticity, is specific to repulsive jamming.

Model. We consider systems of $N=1024$ particles in $d=$ 2 dimensions prepared in a periodic square cell. Each particle has a disk-shaped core of radius $\sigma_{i}$ and an annular shell of thickness $a \sigma_{i}$. We use the standard 50:50 bidisperse mixture with a size ratio of $1.4: 1[3,22]$, and take the core diameter of the small disks as our unit of length. The finite-ranged force between disks is a piecewise function of the overlap $\delta_{i j}=\sigma_{i j}-r_{i j}$, where $\sigma_{i j}=\sigma_{i}+\sigma_{j}$ and $r_{i j}$ is the distance between their centers,

$$
f_{i j}= \begin{cases}k \delta_{i j}, & \delta_{i j} \geqslant-a \sigma_{i j}, \\ -k\left(\delta_{i j}+2 a \sigma_{i j}\right), & -a \sigma_{i j}>\delta_{i j} \geqslant-2 a \sigma_{i j}, \\ 0, & \delta_{i j}<-2 a \sigma_{i j} .\end{cases}
$$

See also Fig. 1(b). This force law is chosen both for its simplicity and for consistency with prior work [14-18,20,21]. Overlapping cores contribute a repulsive springlike interaction with stiffness $k=1$, which fixes our units of stress. Overlap between the outer shells gives an attractive contribution; the parameter $a$ fixes both its range and the maximum tensile force. We use this dimensionless number to characterize attraction strength.

For each attraction strength and packing fraction $\phi$, calculated from the cores, systems are prepared by randomly placing particles in the unit cell and quenching to a local energy minimum using a nonlinear conjugate gradient algorithm [22]. Each system is analyzed with the standard pebble game algorithm [23], which yields a complete set of rigid clusters and redundancies. A rigid cluster is a set of connected particles with no zero-frequency eigenmodes, apart from trivial rigid-body motions. A redundancy is a set of bonds, any one of which can be removed from their cluster without loss of rigidity. For each redundancy there is a 
(a)

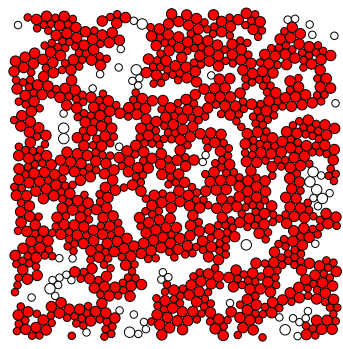

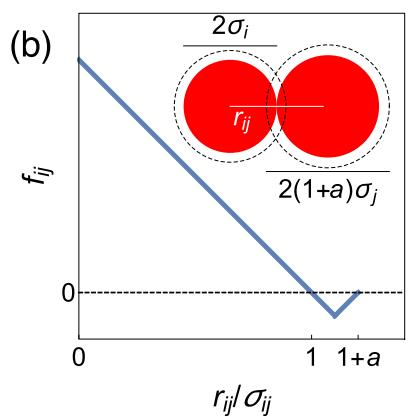

FIG. 1. (a) A periodic packing of sticky disks at $\phi \approx \phi_{c}$. Red disks participate in the spanning rigid cluster. (b) Their force law. The dimensionless parameter $a$ sets the range and strength of the attractive interaction; $a=0.1$ at left.

corresponding state of self-stress (SSS), a balanced configuration of forces compatible with the system's contact network. While redundancies arise naturally in the context of the pebble game, SSS's are more widely discussed in the literature-see, e.g., Refs. [24-29]. We refer to a system as rigid/jammed if it contains a rigid cluster that spans the unit cell. For each jammed state the shear modulus $G$ and bulk modulus $K$ are calculated in the harmonic approximation by inverting the Hessian matrix, as detailed in Refs. [30,31]. Data for each $(a, \phi)$ pair are averaged over 100-1000 samples. Standard errors are smaller than the plotted symbols.

Repulsive systems jam at $\phi=\phi_{c}(0) \approx 0.84177$ [32]. In attractive systems a spanning cluster appears instead at $\phi_{c}(a)=\phi_{c}(0)-\epsilon(a)$, where $\epsilon(a)$ represents the volumetric strain needed to compress the system from $\phi_{c}(0)$ to $\phi_{c}(a)$. In prior work we found that

$$
\epsilon(a) \simeq\left(\frac{a}{a_{0}}\right)^{v},
$$

with $v=0.5$ and $a_{0}=0.80$ [21]; here, we take these values as given. In the same study, we showed that cluster size statistics display finite-size effects when $a<a^{*} \sim 1 / N$. We focus here on $10^{-3} \leqslant a \leqslant 10^{-1}$.

Shear modulus. We first consider the shear modulus $G$ as a function of packing fraction and attraction strength. In Fig. 2(a) one sees that, for each value of $a, G$ vanishes continuously at a $\phi$ that corresponds closely to $\phi_{c}(a)$ determined from rigid cluster percolation (colored arrows). For the smallest attraction strengths in Fig. 1(a) (where $a \simeq a^{*}$ ), the modulus resembles its form in repulsive jamming, $G_{0}(\phi) \Theta\left(\Delta \phi_{0}\right)$, where $\Theta(x)$ is the unit step function and $G_{0} \equiv g_{0} \Delta \phi_{0}^{\mu}$ with $g_{0} \approx 0.22, \Delta \phi_{0} \equiv \phi-\phi_{c}(0)$, and $\mu=1 / 2$ [3]. The initial growth of $G$ becomes shallower with increasing $a$, and any resemblance to the repulsive shear modulus is lost.

We now show that $G(\phi, a)$ can be expressed in terms of a master curve that depends on a single variable $\alpha$. We first define $\mathcal{G}_{ \pm}$as the ratio between the sticky and repulsive shear moduli at the same value of $\left|\Delta \phi_{0}\right|, \mathcal{G}_{ \pm} \equiv G(\phi, a) /\left|G_{0}\right|$. The function has two branches, one for each sign of $\Delta \phi_{0}$. We then motivate $\alpha$ in the following way. Near the sticky jamming point, we expect that $G$ will grow as a power law $G \sim f(a)\left[\phi-\phi_{c}(a)\right]^{\psi}$. [The prefactor $f(a)$ will be discussed below.] Deep in the sticky jammed phase, tensile forces
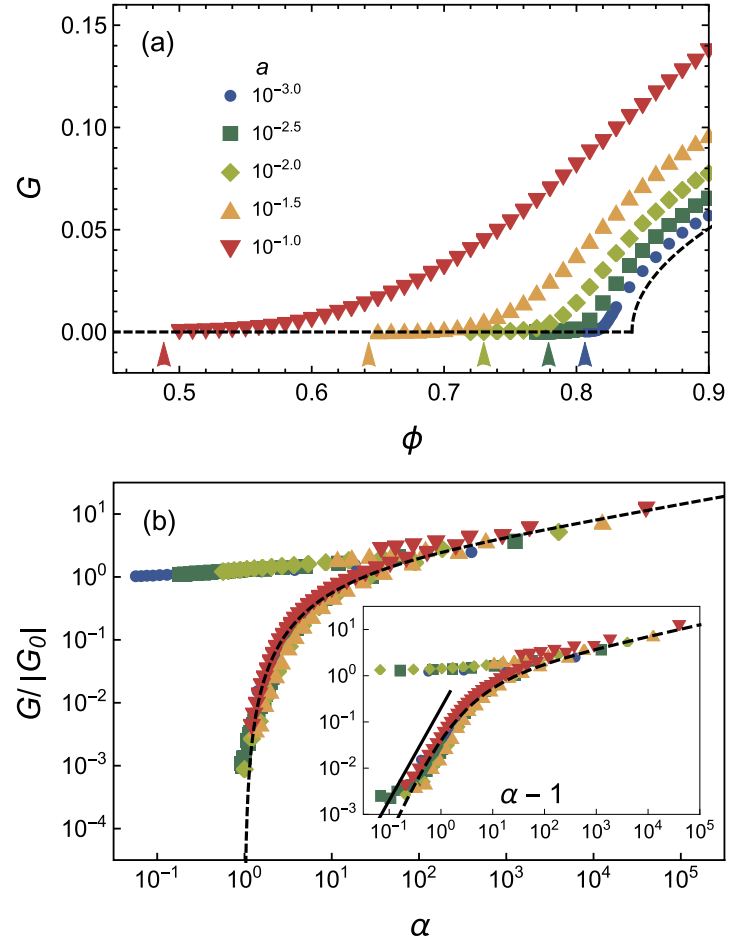

FIG. 2. (a) The shear modulus of sticky packings at varying attraction strengths $a$. The legend applies to this and all subsequent figures. The dashed curve is $G$ for $a=0$. Arrows indicate $\phi_{c}(a)$ according to Eq. (2). (b) Data collapse of the shear modulus according to Eq. (4). Inset: The same data plotted vs $\alpha-1$. The dashed curves depict Eq. (6) with $\psi=2.5$. The solid line has slope 2 .

are rare and packings resemble purely repulsive systems. We therefore anticipate that $G \simeq G_{0} \sim \Delta \phi_{0}^{\mu}$ will be recovered. The crossover between these two scaling relations must be governed by the distances $\phi-\phi_{c}(a)=\Delta \phi_{0}+\epsilon(a)$ and $\left|\Delta \phi_{0}\right|$. Their ratio is a function of

$$
\alpha \equiv\left(\frac{\epsilon}{\left|\Delta \phi_{0}\right|}\right)^{1 / v} \simeq \frac{a / a_{0}}{\left|\Delta \phi_{0}\right|^{1 / v}} .
$$

For later convenience we have defined $\alpha$ so as to be linear in $a$. By construction the jamming point is at $\alpha_{c}=1$ and jammed states have $\alpha \geqslant 1$. Finally, we make the scaling ansatz that $\mathcal{G}_{ \pm}$ is a function of $\alpha$,

$$
\frac{G(\phi, a)}{\left|G_{0}\right|}=\mathcal{G}_{ \pm}(\alpha) .
$$

We emphasize that Eq. (4) depends only on known quantities: the repulsive jamming point $\phi_{c}(0)=0.84177$ [32]; the repulsive shear modulus (exponent $\mu=0.5$ ) [3]; and the shift in the sticky jamming point $\epsilon$ determined from rigid cluster analysis (exponent $v=0.5$ ) [21]. Equation (4) is therefore a prediction without any free parameters.

Figure 2(b) verifies the data collapse predicted in Eq. (4). The upper branch $\mathcal{G}_{+}$approaches unity when $\alpha$ vanishes, i.e., the modulus for repulsive jamming is recovered. The lower branch $\mathcal{G}_{-}$vanishes for states below $\alpha \approx 1$, indicative of unjamming. (The same data are plotted versus $\alpha-1$ in the inset.) Some states do exist slightly below $\alpha=1$; we attribute this to finite-size effects, which smear out $\phi_{c}(a)$ [21]. It is 
apparent that there is also a third scaling regime, in which both branches scale as $\mathcal{G}_{ \pm} \sim \alpha^{\Delta}$ for some positive exponent $\Delta$. This expression describes the shear modulus when $\phi \approx$ $\phi_{c}(0)$. Since $G$ remains finite at $\phi_{c}(0)$ when $a$ is nonzero, any dependence on $\Delta \phi_{0}$ must be subdominant. This requirement is only satisfied if $\Delta=\mu \nu=0.25$.

We now apply data fitting to the master curve in Fig. 2(b) to estimate the exponent $\psi$ that describes the initial growth of $G$ above the sticky jamming point. As stated above, we anticipate

$$
G \simeq f(a)\left[\phi-\phi_{c}(a)\right]^{\psi},
$$

for some $\psi>0$. The prefactor $f(a)$ is present because there is no reason to forbid it. We now assume $f \simeq c_{G} a^{-\omega}$ to leading order. Importantly, the exponent $\omega$ is not free; it is only compatible with Eq. (4) if $\omega=v \psi-\Delta$. Hence if the shear modulus obeys Eq. (5) near $\phi_{c}(a)$, the lower branch of the scaling function must scale as

$$
\mathcal{G}_{-} \simeq c_{G} \alpha^{\Delta}\left[1-\alpha^{-v}\right]^{\psi}
$$

sufficiently close to $\alpha=1$. Equation (6) has a crossover from $\mathcal{G}_{-} \sim(\alpha-1)^{\psi}$ as $\alpha \rightarrow 1^{+}$to $\mathcal{G}_{-} \sim \alpha^{\Delta}$ when $\alpha \rightarrow \infty$. Therefore we can estimate $\psi$ in two ways: first, by fitting $(\alpha-1)^{\psi}$ to data at small $\alpha-1$, or, second, by fitting a wider range of data with Eq. (6). The first approach is rigorous and preferable when many decades of data in $\alpha-1$ are available below the crossover. As our data cover less than two decades, fitting to $(\alpha-1)^{\psi}$ can erroneously lower the estimate of $\psi$. The second approach "knows about" the crossover and so allows one to fit to more data. But it is also less rigorous, because it is not a priori clear how far from $\alpha=1$ Eq. (6) holds. Recall that $\mathcal{G}_{-} \sim \alpha^{\Delta}$ as $\alpha \rightarrow \infty$ is required as a consequence of the scaling collapse in Fig. 2(b). As Eq. (6) matches this form, we attempt to fit it to the entire lower branch. One could write down other functions that cross over between the same power-law scalings as Eq. (6), and this choice may influence the fitted value of $\psi$. The result of the first approach is depicted by the solid curve in Fig. 2(b). We find $\psi=2.0$ (hence $\omega=0.75$ ). Fitting the lower branch to Eq. (6) yields instead $\psi=2.5(\omega=1.0)$, with $c_{G}=0.81$ - see the dashed curve. We therefore expect that the critical exponent is at least 2.0 and likely in the interval $\psi \in[2.0,2.5]$. We emphasize that $\psi$ is clearly larger than the corresponding exponent $\mu$ in repulsive packings, which signals the distinct mechanical character of sticky jammed matter.

Bulk modulus. Next, we consider the bulk modulus $K$ as a function of packing fraction while varying the attraction strength, as shown in Fig. 3(a). The sticky modulus is always continuous, with a steep slope near $\phi_{c}(0)$ for the smallest values of $a$. For context, we recall that for purely repulsive particles with harmonic interactions, the repulsive bulk modulus vanishes discontinuously at $\phi_{c}(0)$ [Fig. 3(a), dashed curve], unlike the repulsive shear modulus. The jump has been shown to result from self-organization into a state that can support isotropic stress without tensile forces [33,34]. Closely related systems such as randomly pruned spring networks, which do not self-organize and do permit tensile forces, have a continuous $K$ that vanishes with the same exponent as $G$ [35]. Tensile forces are also present in sticky packings, which suggests the latter scenario may be relevant near $\phi_{c}(a)$.
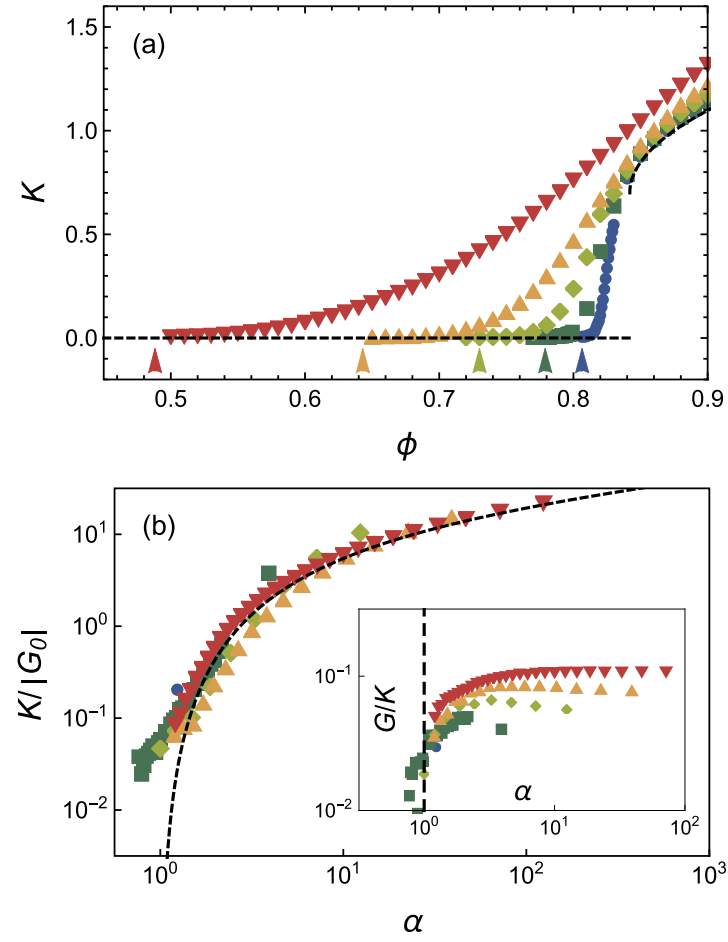

FIG. 3. (a) The bulk modulus of sticky disk packings at varying attraction strengths $a$. The dashed curve shows $K$ for $a=0$. (b) The rescaled bulk modulus for $\phi \leqslant 0.81$ plotted vs $\alpha$. The dashed curve depicts $11.1 c_{G} \alpha^{\Delta}\left[1-\alpha^{-v}\right]^{\psi}$ with $c_{G}=0.81$ and $\psi=2.5$, as in Fig. 2(b). Inset: The ratio of shear to bulk modulus, also for $\phi \leqslant$ 0.81 .

It is therefore reasonable to ask if the bulk modulus and the shear modulus have the same scaling near the sticky jamming point, i.e., if $K \sim a^{-\omega}\left[\phi-\phi_{c}(a)\right]^{\psi}$ with the same exponent $\psi$ (and hence $\omega$ as well). As a first check, we focus on packing fractions $\phi \leqslant 0.81$, well below $\phi_{c}(0)$, and rescale $K$ in the same way we rescaled $G$ in Fig. 2(b); namely, we plot $K /\left|G_{0}\right|$ vs $\alpha$. (Note that while we choose to divide by $\left|G_{0}\right|$, any multiple of $\left|\Delta \phi_{0}\right|^{\mu}$ would work.) We then look to see if, as in Eq. (6), the rescaled data scale as $\alpha^{\Delta}\left[1-\alpha^{-v}\right]^{\psi}$ for some range of $\alpha$. This test is shown in Fig. 3(b). While there is more scatter than in Fig. 2(b), the data are indeed reasonably described by the same scaling relation as $G$ using $\psi=2.5$. This result should be interpreted cautiously, however, as a plot of the ratio $G / K$ [Fig. 3(b), inset] reveals additional subtlety. If $G$ and $K$ vanish with the same exponent, each curve should approach a finite value as $\alpha \rightarrow 1^{+}$. Instead, the data turn downwards in the vicinity of $\alpha=1$. This could be due to a bulk modulus exponent $\psi^{\prime}$ that is slightly smaller than the shear exponent $\psi$. Alternatively, it may be a finite-size effect.

Coordination and redundancies. The coordination of a network characterizes its structure and plays a fundamental role in theories of the moduli in repulsive jamming, e.g., Refs. [33,36]. It is therefore useful to identify correlations between coordination and packing fraction.

Let us first recall the main result of Maxwell-Calladine counting, which relates degrees of freedom, constraints on motion, floppy modes, and redundancies in a network of nodes and bonds (viz., particles and contacts) [24]. It states 

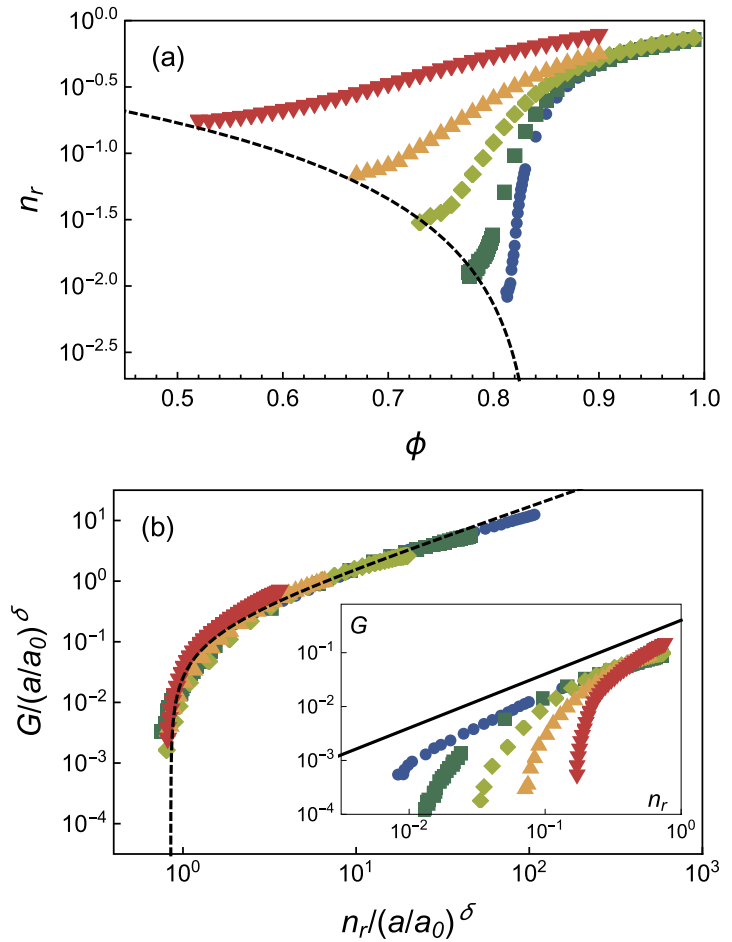

FIG. 4. (a) The redundancy density $n_{r}$ plotted as a function of packing fraction for varying attraction strengths. The dashed curve shows $n_{c} \sim\left[\phi_{c}(0)-\phi_{c}(a)\right]^{\delta / v}$. (b) Collapse of the shear modulus with $n_{r}$ when both are rescaled with $a^{\delta}$. $G \sim n_{r}-n_{c}$ (dashed curve) is plotted for comparison. Inset: Unscaled data. The solid line has slope 1 .

that $d-\frac{1}{2} z=n_{f}-n_{r}+O(1 / N)$, where $z$ is the coordination averaged over nodes, and $n_{f}$ and $n_{r}$ are the numbers of floppy modes and redundancies per node, respectively. Neglecting the $O(1 / N)$ correction and applying this relation to a spanning rigid cluster, which has no floppy modes, gives $z=z_{\text {iso }}+2 n_{r}$, where $z_{\text {iso }} \equiv 2 d$ is the Maxwell isostatic value. Therefore creating new contacts, e.g., by compression, also introduces an equal number of redundancies. While Maxwell-Calladine counting places no further constraints on $n_{r}$, it is an empirical fact that $n_{r}$ vanishes at the jamming point in repulsive systems [3,37-39], i.e., $z_{c}(a=0)=z_{\text {iso }}$. In fact, $z=z_{\text {iso }}$ is often used as a criterion for jamming. Given this context, in Fig. 4(a) we plot $n_{r}$ vs $\phi$ for varying $a$. Unlike repulsive systems, $n_{r}$ remains finite as $\phi$ approaches $\phi_{c}(a)$, indicating that the spanning cluster is overconstrained, $z_{c}(a)>z_{\text {iso }}$. Hence Maxwell's isostatic value does not signal rigidity percolation in sticky systems.

In order to quantify the critical redundancy density $n_{c}(a) \equiv$ $\left.n_{r}(a, \phi)\right|_{\phi=\phi_{c}(a)}$, we investigate the point where the shear modulus vanishes in a plot of $G$ vs $n_{r}$, as shown in the inset of Fig. 4(b). We have verified that the bulk modulus also vanishes at the same redundancy density (not shown). We seek to collapse the data by plotting $G /\left(a / a_{0}\right)^{\delta}$ vs $n_{r} /\left(a / a_{0}\right)^{\delta}$. The same exponent $\delta$ must appear on each axis to ensure that the known form for repulsive systems, $G \sim n_{r}[3,33]$, is recovered when $a \rightarrow 0$. We find good data collapse for $\delta=0.75$, with the master curve vanishing at a value $n_{0} \approx 0.85$ on the abscissa [Fig. 4(b), main panel]. It follows that $n_{c} \simeq n_{0}\left(a / a_{0}\right)^{\delta}$, and that the excess coordination at the sticky jamming point is $z_{c}(a)-z_{\text {iso }}=2 n_{c} \sim\left[\phi_{c}(0)-\phi_{c}(a)\right]^{\delta / v}$ [Fig. 4(a), dashed curve]. Above the sticky jamming point, the expression $G \sim$ $n_{r}-n_{c}$ represents a natural generalization of the repulsive case and provides a reasonable fit to our data [dashed curve, Fig. 4(b), main panel]. In other words, $G$ grows in proportion to the number of excess redundancies compared to the spanning cluster at percolation.

Discussion. We have shown that sticky jamming differs from repulsive jamming in three distinct ways. While the shear modulus in repulsive packings vanishes continuously with a critical exponent $\mu=1 / 2$, in sticky jamming the exponent $2.0 \lesssim \psi \lesssim 2.5$ is much larger. The bulk modulus in sticky systems vanishes continuously and apparently in proportion to $G$, unlike repulsive jamming where it has a jump. Also, redundancies persist at the sticky jamming point, with number density $n_{c} \sim a^{\delta}$ and $\delta \approx 0.75$. In contrast, $n_{c}=0$ at repulsive jamming.

The mechanical and structural properties identified here represent a challenge to existing theories of elasticity in marginal solids $[33,34,36,40]$. A successful theory should predict the values of the exponents $\psi, \delta$, and $v$, each of which remain empirical. Effective medium theories for marginal elastic solids predict both $G$ and $K$ to vanish continuously [40]; however, they do not successfully account for the repulsive case [35]. While there is a successful theory of elasticity in repulsive jammed solids [33], a straightforward generalization of its results would predict both $G$ and $K$ are discontinuous at sticky unjamming due to the presence of redundancies. More qualitatively, we are not aware of any theory that predicts unjamming with $n_{c}>0$, and hence $z>z_{\text {iso }}$.

We see several directions for future work. Most obviously, the measurement of the shear exponent $\psi$ should be improved. Similarly, one should determine more conclusively whether the same exponent describes the bulk modulus. Both tasks can be achieved with a data set that accesses smaller values of $\alpha-1$ and larger system sizes. This will also permit an analysis of finite-size effects and corrections to scaling. Next, simulations in three dimensions are needed. While we expect critical scaling to persist, the exponents $\psi, \delta$, and $v$ may differ, as is the case for other exponents near sticky jamming [20]. In addition, the force law in Eq. (1) is particularly simple, with just one parameter $a$; untangling the role of, e.g., the maximum tensile force and the range of the interaction will facilitate comparisons to experiment. Finally, it is natural to ask how sticky systems respond to oscillatory shear, which would build a bridge between the present work, viscoelasticity in repulsive jamming [31,41-43], and steady shear flow in sticky systems $[17,18]$.

Acknowledgments. We acknowledge financial support from the Netherlands Organization for Scientific Research (NWO). This work was sponsored by NWO Physical Sciences through the use of supercomputer facilities. 
[1] A. J. Liu and S. R. Nagel, Nature (London) 396, 21 (1998).

[2] D. J. Durian, Phys. Rev. Lett. 75, 4780 (1995).

[3] C. S. O’Hern, L. E. Silbert, A. J. Liu, and S. R. Nagel, Phys. Rev. E 68, 011306 (2003).

[4] M. van Hecke, J. Phys.: Condens. Matter 22, 033101 (2010).

[5] L. Bécu, S. Manneville, and A. Colin, Phys. Rev. Lett. 96, 138302 (2006).

[6] I. Jorjadze, L.-L. Pontani, K. A. Newhall, and J. Brujić, Proc. Natl. Acad. Sci. U.S.A. 108, 4286 (2011).

[7] I. Golovkova, L. Montel, E. Wandersman, T. Bertrand, A. M. Prevost, and L.-L. Pontani, Soft Matter 16, 3294 (2020).

[8] S. Cox, A. Kraynik, D. Weaire, and S. Hutzler, Soft Matter 14, 5922 (2018).

[9] S. Herminghaus, Adv. Phys. 54, 221 (2005).

[10] P. C. Møller and D. Bonn, Europhys. Lett. 80, 38002 (2007).

[11] A. Singh, V. Magnanimo, K. Saitoh, and S. Luding, Phys. Rev. E 90, 022202 (2014).

[12] A. Hemmerle, M. Schröter, and L. Goehring, Sci. Rep. 6, 35650 (2016).

[13] E. Koos and N. Willenbacher, Science 331, 897 (2011).

[14] D. Head, Eur. Phys. J. E 22, 151 (2007).

[15] W. Zheng, H. Liu, and N. Xu, Phys. Rev. E 94, 062608 (2016).

[16] P. Chaudhuri, L. Berthier, and L. Bocquet, Phys. Rev. E 85, 021503 (2012).

[17] E. Irani, P. Chaudhuri, and C. Heussinger, Phys. Rev. Lett. 112, 188303 (2014).

[18] E. Irani, P. Chaudhuri, and C. Heussinger, Phys. Rev. E 94, 052608 (2016).

[19] G. Katgert, B. P. Tighe, and M. van Hecke, Soft Matter 9, 9739 (2013).

[20] G. Lois, J. Blawzdziewicz, and C. S. O’Hern, Phys. Rev. Lett. 100, 028001 (2008).

[21] D. J. Koeze and B. P. Tighe, Phys. Rev. Lett. 121, 188002 (2018).

[22] D. J. Koeze, D. Vågberg, B. B. Tjoa, and B. P. Tighe, Europhys. Lett. 113, 54001 (2016).
[23] D. J. Jacobs and M. F. Thorpe, Phys. Rev. Lett. 75, 4051 (1995).

[24] S. Pellegrino and C. R. Calladine, Int. J. Solids Struct. 22, 409 (1986).

[25] S. Alexander, Phys. Rep. 296, 65 (1998).

[26] J.-N. Roux, Phys. Rev. E 61, 6802 (2000).

[27] B. P. Tighe and T. J. H. Vlugt, J. Stat. Mech.: Theory Exp. (2011) P04002.

[28] E. Lerner, G. Düring, and M. Wyart, Proc. Natl. Acad. Sci. USA 109, 4798 (2012).

[29] J. Paulose, A. S. Meeussen, and V. Vitelli, Proc. Natl. Acad. Sci. USA 112, 7639 (2015).

[30] C. E. Maloney and A. Lemaître, Phys. Rev. E 74, 016118 (2006).

[31] B. P. Tighe, Phys. Rev. Lett. 107, 158303 (2011).

[32] D. Vågberg, P. Olsson, and S. Teitel, Phys. Rev. E 83, 031307 (2011).

[33] M. Wyart, Ann. Phys. 30, 1 (2005).

[34] M. Merkel, K. Baumgarten, B. P. Tighe, and M. L. Manning, Proc. Natl. Acad. Sci. USA 116, 6560 (2019).

[35] W. G. Ellenbroek, Z. Zeravcic, W. van Saarloos, and M. van Hecke, Europhys. Lett. 87, 34004 (2009).

[36] M. Schlegel, J. Brujic, E. Terentjev, and A. Zaccone, Sci. Rep. 6, 18724 (2016).

[37] C. P. Goodrich, A. J. Liu, and S. R. Nagel, Phys. Rev. Lett. 109, 095704 (2012).

[38] S. Dagois-Bohy, B. P. Tighe, J. Simon, S. Henkes, and M. van Hecke, Phys. Rev. Lett. 109, 095703 (2012).

[39] C. P. Goodrich, S. Dagois-Bohy, B. P. Tighe, M. van Hecke, A. J. Liu, and S. R. Nagel, Phys. Rev. E 90, 022138 (2014).

[40] S. Feng, M. F. Thorpe, and E. Garboczi, Phys. Rev. B 31, 276 (1985).

[41] K. Baumgarten and B. P. Tighe, Soft Matter 13, 8368 (2017).

[42] S. Dagois-Bohy, E. Somfai, B. Tighe, and M. van Hecke, Soft Matter 13, 9036 (2017).

[43] K. Saitoh, T. Hatano, A. Ikeda, and B. P. Tighe, Phys. Rev. Lett. 124, 118001 (2020). 